\title{
Obesity turns the hepatic epigenetic clock forward
}

Acceleration of the 'epigenetic clock' in the liver could be a molecular link between obesity and increased risk of some agerelated diseases, suggests a new study published in the Proceedings of the National Academy of Sciences of the USA.

Although individuals with obesity are widely recognised to be at increased risk of developing age-related diseases such as cancer, type 2 diabetes mellitus and heart disease, the molecular underpinnings that

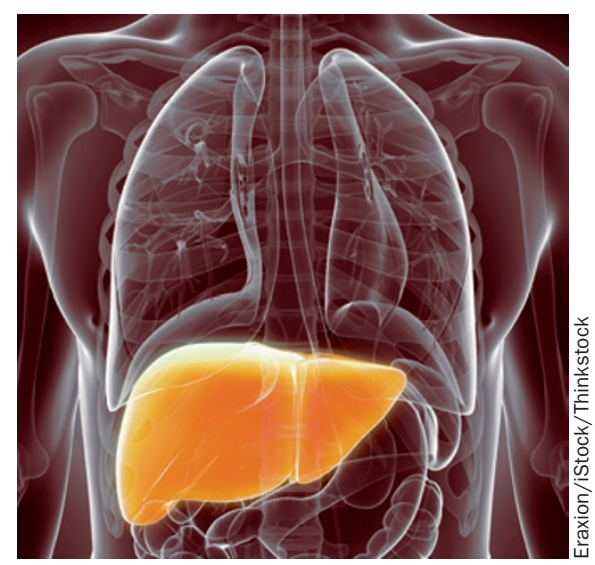

drive these pathologies in the context of obesity remain largely unknown.

Lead author Steve Horvath, from the University of California Los Angeles, previously published research describing a novel biomarker for ageing-the epigenetic clock-that was developed on the basis of changes in DNA methylation (DNAm) patterns. Now, Horvath has partnered with an international team of experts to show that the epigenetic clock is irreversibly accelerated in the livers of patients with obesity, which possibly explains why these individuals are at increased risk of developing comorbidities such as insulin resistance and liver cancer.

The researchers used DNAm datasets of blood, liver, adipose tissue and muscle tissue, paired with data on chronological age, BMI and sex, to assess the effects of obesity on molecular ageing in these tissues. As DNAm age is strongly correlated with chronological age, acceleration of molecular ageing was determined using a linear model that regressed mDNA age on chronological age. Thus, tissues with positive age acceleration seemed older than expected according to chronological age; conversely, tissues with negative accelerated ageing seemed younger than expected.

Surprisingly, the liver was the only tissue in which a correlation between acceleration of ageing and BMI was shown. "On average, the increase in age of liver tissue was 3.3 years for each 10 units of BMI," explains Horvath. Moreover, when samples of patients who had received metabolic surgery were analyzed 9 months after treatment, their livers showed no signs of reversal of ageing, despite the loss of weight.

The researchers are now interested in understanding the mechanisms that drive ageing in hepatocytes, in addition to testing the practicability of using the epigenetic age of the liver as a prognostic marker.

\section{Jennifer Sargent}

Original article Horvath, S. et al. Obesity accelerates epigenetic aging of human liver. Proc. Natl Acad. Sci. USA doi:10.1073/pnas.1412759111 